\title{
On the unstable mode merging of gravity-inertial waves with Rossby waves
}

\author{
J. F. McKenzie \\ Department of Mathematics, Statistics and Physics, Durban University of Technology, Durban, South Africa \\ School of Mathematical Sciences, University of KwaZulu-Natal, Durban, South Africa \\ CSPAR, University of Alabama, Huntsville, USA
}

Received: 30 May 2011 - Revised: 15 July 2011 - Accepted: 2 August 2011 - Published: 19 August 2011

\begin{abstract}
We recapitulate the results of the combined theory of gravity-inertial-Rossby waves in a rotating, stratified atmosphere. The system is shown to exhibit a "local" (JWKB) instability whenever the phase speed of the lowfrequency-long wavelength westward propagating Rossby wave exceeds the phase speed ("Kelvin" speed) of the high frequency-short wavelength gravity-inertial wave. This condition ensures that mode merging, leading to instability, takes place in some intermediate band of frequencies and wave numbers. The contention that such an instability is "spurious" is not convincing. The energy source of the instability resides in the background enthalpy which can be released by the action of the gravitational buoyancy force, through the combined wave modes.
\end{abstract}

Keywords. Meteorology and atmospheric dynamics (Ocean-atmosphere interactions)

\section{Summary of the problem}

McKenzie (2011, 2009) showed that the mode merging of westward propagating gravity-inertial waves with Rossby waves can lead to instability of the wave system describing small, (linearized), perturbations of the equations of motion in a stratified, rotating atmosphere in the $\beta$-plane approximation. From a wave dynamics viewpoint the instability arises if the westward phase speed of the "low frequencylong wavelength" Rossby wave exceeds the phase speed of the "high frequency-short wavelength" gravity-inertial mode so as to ensure that they interact ("mode merging") at some

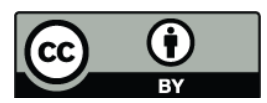

Correspondence to: J. F. McKenzie (mckenziej@ukzn.ac.za) intermediate range of frequencies and wave numbers (but see Fig. 1 for a clear picture). This intuitive prediction is vindicated by an analysis of the local dispersion equation appropriate to mid-latitudes, or JWKB waves packets propagating in the body of the atmosphere (ocean), and is presented in the next section.

Paldor (2010) claims that such an instability is "spurious". In Sect. 3 we address his arguments and conclude that they are not convincing. We conclude that the energy reservoir which may be tapped to drive the instability resides in the enthalpy of the background stratified atmosphere. The gravitational buoyancy force liberates this enthalpy through the action of the combined wave modes whenever the stability parameter $m>1$. This, apparently new, instability may play an important role in planetary atmospheric dynamics.

\section{The dispersion equations and the instability condition}

The governing equations for small amplitude perturbations in a stratified, rotating atmosphere (e.g. McKenzie, 2009, 2011) yield a wave equation for the system, which for Fourier plane wave solutions of the form $Q(y) \exp i\left(\omega t-k_{x x}-k_{z z}\right)$ for $q_{y}$ the perturbation northward $(y)$, mass flux $\left(\rho_{0} u_{y}\right)$, reduces to the following second order ordinary differential equation for the latitudinal structure $Q(y)$,

$\frac{d^{2} Q}{d y^{2}}=-K^{2} Q$,

in which the square of the "wave number" is given by

$K^{2} \equiv \frac{\left(\omega^{2}-f^{2}\right) k_{z}^{2}}{\left(N^{2}-\omega^{2}\right)}-k_{x}^{2}-\beta \frac{k_{x}}{\omega}$

Here $f=f_{0}+\beta y\left(f_{0}=2 \Omega \sin \theta_{0}, \beta=2 \frac{\Omega}{a} \cos \theta_{0}\right)$ in the $\beta$ plane constructed tangent to the planet of radius $a$, at latitude

Published by Copernicus Publications on behalf of the European Geosciences Union. 
$\theta_{0}$ with $y$ measuring distance northward. The Vaisala-Brunt, or bounce, frequency, follows from the condition of adiabatic flow, and is given by (Lighthill, 1980; Eckart, 1960)

$N^{2}=-g\left(\frac{\rho_{0}^{\prime}(z)}{\rho_{0}(z)}+\frac{g}{c_{0}^{2}}\right)$

in which $\rho_{0}(z)$ is the background atmosphere density stratified according to

$\frac{d p_{0}}{d z}=-\rho_{0} g$

and $c_{0}=\sqrt{\gamma p_{0} / \rho_{0}}$ is the sound speed. High frequency acoustic waves have been filtered out of the analysis using the Boussinesq approximation in the adiabatic flow condition and continuity equation.

The latitude structure $Q(y)$ is "oscillatory" if $K^{2}>0$, and evanescent if $K^{2}<0$. Therefore either radiation or evanescent conditions may be imposed "far" from the $\beta$-plane latitude to eliminate unphysical solutions. Indeed, if one wishes artificial rigid zonal walls erected at given latitudes may be imposed which would yield wave-guide type eigenvalues for $K$, but this is neither realistic nor illuminating (Paldor et al., 2009). However if $y \ll f_{0} / \beta$ so that $f$ and hence $K$, may be regarded as nearly constant Eq. (1) is satisfied by $Q(y) \propto \exp \left(\mp i k_{y} y\right)$, and one obtains the "mid-latitude" dispersion equation (Pedolsky, 2003), $k_{y}^{2}=K^{2}$, which may cast in the diagnostic form,

$\omega\left(\omega^{2}-\omega_{i}^{2}\right)=\beta V^{2} k_{x}$

$\omega_{i}^{2} \equiv f_{0}^{2}+V^{2}\left(k_{x}^{2}+k_{y}^{2}\right)$

$V^{2} \equiv \frac{\left(N^{2}-\omega^{2}\right)}{k_{z}^{2}}$

This is also the "local " dispersion equation in which $f=$ $f_{0}+\beta y$, associated with JWKB type solutions of the form

$Q(y) \propto \frac{1}{k_{y}^{1 / 2}} \exp \left( \pm i \int k_{y} d y\right)$

in which $k_{y}^{2}=K^{2}$, which apply in the "slowly varying medium" limit or "short wavelength" approximation. These approximate solutions describe JWKB wave packets propagating in the body of the atmosphere ("internal waves"), located well within any boundaries and therefore boundary terms (conditions) are surely irrelevant to their properties. The dispersion Eq. (5) has three roots for the wave frequency $\omega$; the two "higher" frequency roots correspond to gravity-inertial waves, which propagate both eastward and westward, whilst the remaining "low frequency" root describes the westward (and northward) propagating Rossby wave. The approximate dispersion equation for these roots assume the well known forms,

$$
\begin{aligned}
\omega^{2} \doteqdot \omega_{i}^{2} \equiv\left(f^{2}+V^{2}\left(k_{x}^{2}+k_{y}^{2}\right)\right) & \text { gravity-inertial } \\
\omega \doteqdot-\frac{\beta V^{2} k_{x}}{\omega_{i}^{2}}=\frac{-\beta k_{x}}{\frac{f^{2}}{V^{2}}+k_{x}^{2}+k_{y}^{2}} & \text { Rossby }
\end{aligned}
$$

Observe that long wavelength-low frequency, Rossby waves propagate westward at the speed $\beta V^{2} / f^{2}$, whereas westward propagating high frequency-short wavelength gravity-inertial waves do so at speed $V$. A wave dynamist would immediately expect wave coupling (or mode merging) to take place at some intermediate band of frequencies and wave numbers if the former exceeds the latter,

$\frac{\beta V^{2}}{f^{2}}>V \quad$ or $\quad \frac{\beta V}{f^{2}}>1$

As we shall now see this is precisely the condition which leads to the appearance of complex conjugate roots, for $\omega$ in Eq. (5), corresponding to instability. With

$\bar{\omega}=\frac{\omega}{f}$

and

$\bar{k}=k \frac{V}{f}$

the normalized dispersion equation may be written

$\bar{\omega}\left(\bar{\omega}-\bar{\omega}_{i}^{2}\right)=m \bar{k}$

$\bar{\omega}_{i}^{2}=1+\bar{k}^{2}, \quad \bar{k}=\left(\bar{k}_{x}, \bar{k}_{y}\right)=\bar{k}(\cos \phi, \sin \phi)$

and the stability parameter is defined as

$m=\frac{\beta V}{f^{2}} \cos \phi$.

It follows in a straightforward fashion (but see McKenzie, 2009, 2011 for details) that the westward roots coalesce when $m=1$, becoming complex conjugate when

$m>1$

in agreement with relation (Eq. 8). The diagnostic diagram is shown in Fig. 1 for three values of $m(\lesseqgtr) 1$. The case $m=1$ represents "marginal stability" in which the system is teetering on the brink, so to speak. The unstable growth rates $\gamma$ for $m>1$ have been given elsewhere (McKenzie, 2009) but are of the order of day or so in the middle of the unstable band for $m=1.5$.

The case $m=1$ is special with the $(\bar{\omega}, \bar{k})$ curves and the locus of infinite and zero group velocities all intersecting at a common point. The equatorial case $\left(\theta_{0}=0\right)$ is also special since $f_{0} \rightarrow 0$ and a wave-guide type of the system develops. The latitudinal structure can then be expressed in Hermite polynomials, associated with which are eigenvaluesdispersion equations (see for example Cane and Sarachik, 1976; Moore and Philander, 1977). These eigenvalues have precisely the same form as the "mid-latitude" or local dispersion equation, except that the gravity-inertial frequency $\omega_{i}^{2}$ in Eq. (5) is replaced by

$\omega_{i}^{2}=(2 n+1) \beta V+V^{2} k_{x}^{2}, \quad n=0,1,2, \ldots$. 


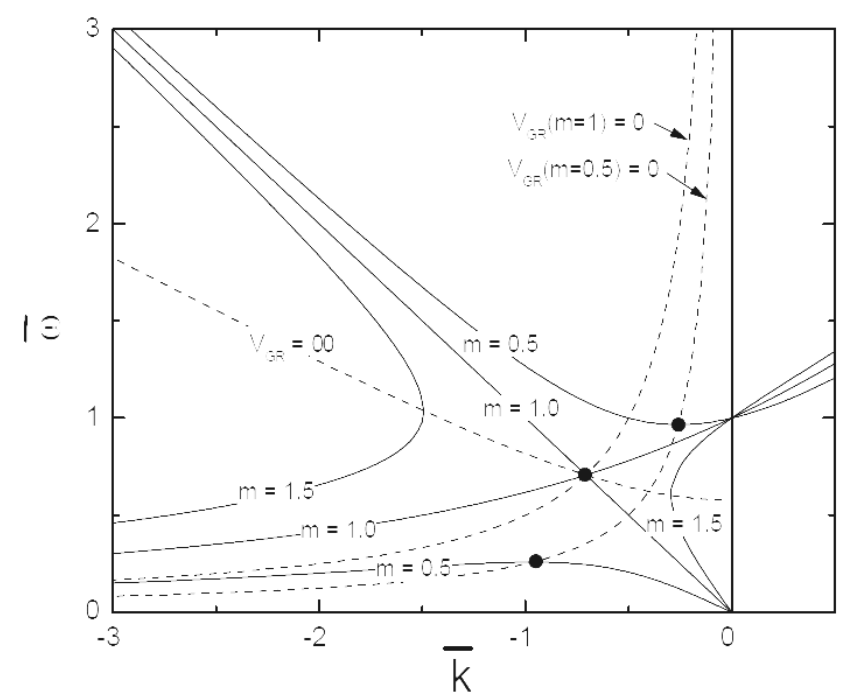

Fig. 1. The "mid-latitude"/JWKB dispersion $(\bar{\omega}, \bar{k})$ curves for three values of the stability parameter $m: m<1(0.5)$ stable, $m=$ 1, marginally stable, $m>1(1.5)$ unstable in the intermediate frequency-wave number band lying between the intersection of the $(\bar{\omega}, \bar{k})$ curves and the locus of infinite group velocity $V_{\mathrm{gr}}=\infty$.

On normalizing $\omega$ to $\sqrt{\beta V}$ and $k_{x}$ to $\sqrt{V / \beta}$ the equatorial dispersion equations take the form

$\bar{\omega}\left(\bar{\omega}-\left(2 n+1+\bar{k}^{2}\right)\right)=\bar{k}, \quad n=0,1,2, \ldots$.

The dispersion curves are shown in Fig. 2 (for $n=0,1,2$ ).

Note that the fundamental, $n=0$, corresponds exactly to the mid-latitude case for $m=1$ ! The root $\bar{\omega}=-\bar{k}$, representing the westward Kelvin wave, factors out, leaving the mixed Yanai mode

$\bar{\omega}(\bar{\omega}-\bar{k})=1$.

This special westward Kelvin mode $(\bar{\omega}=-\bar{k})$ is normally discarded on the basis of evanescent requirements at large $y$. However it is interesting and important to note that this system is strictly speaking, marginally stable. Instability does not arise in this case because the westward speed of any equatorial Rossby wave cannot exceed $V$. The "mid-latitude" instability condition $m>1$, translates into the regions beneath the curves in the $\left(\theta_{0}, M\right)$ plane defined by $m=1$. These are shown in Fig. 3 for various prescribed values of the latitudinal wave number and also for an evanescent case $\left(k_{y}^{2}=-4\right)$.

\section{The wave-energy equation}

Paldor (2010) claims that the unstable solutions associated with the "mid-latitude"/JWKB dispersion equation are "spurious". His arguments are based on an admixture of "a global energy integral" for the system, combined with a discussion of the eigenvalue-dispersion relation (a cubic equation), and the existence of Poisson brackets and a Hamiltonian, for the

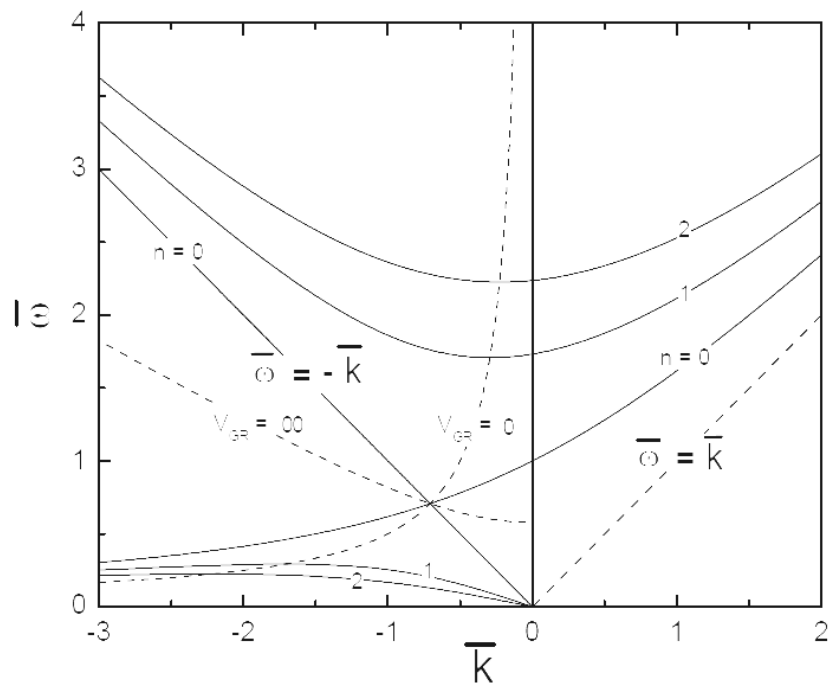

Fig. 2. The equatorial dispersion curves for $n=0,1,2$. Note that the $n=0$ curves, corresponding exactly to the $m=1$ curves of the mid-latitude case, all intersect at the intersection between the locii of zero and infinite group velocity. This case is "marginally stable".

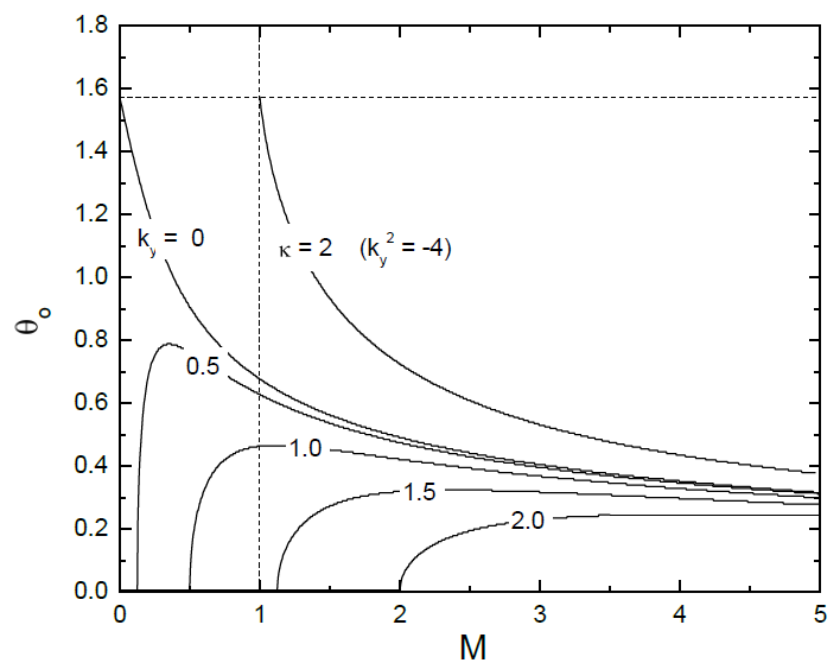

Fig. 3. The $\left(\theta_{0}, M\right)$ parameter space in which the region below the curves given by $m=1$ is unstable.

system (which properties provide a "deeper meaning" of energy conservation). Paldor contends that these considerations preclude the possibility of an instabilty.

In the first place my work is not based on the shallow water theory used by Paldor Paldor (2010). There is a formal equivalence between the two developments in which my "Kelvin" speed $V$ (Eq. 5) may be replaced by the shallow water speed $\sqrt{g h}$ in an ocean of depth $h$. The standard set of equations of motion which I use readily yield the wave energy-exchange equation

$\frac{\partial E}{\partial t}+\operatorname{div} \underline{F}=\frac{p_{e} u_{z}}{H}$ 
in which $E=\frac{1}{2}\left(\rho_{0} u^{2}+V_{s}^{2} \rho_{e}^{2} / \rho_{0}\right)$, and $\underline{F}=p_{e} \underline{u}$ are respectively the wave energy density per unit volume and the wave energy flux. Here $\underline{u}=\left(u_{x}, u_{y}, u_{z}\right)$ is the perturbed fluid velocity, $p_{e}$ and $\rho_{e}$ are the associated pressure and density perturbations, and $V_{s}^{2}=g^{2} / N^{2}$ so that the second term in $E$ is the thermobaric wave energy (Eckart, 1960) and the first the kinetic energy. The term on the right hand side represents an "exchange" with the inhomogeneous background whose density has a scale height $H\left(\left(\rho_{0}^{\prime} / \rho_{0}\right)^{-1}\right)$. (A similar term of the form $\rho_{0} u_{x} u_{y} d V_{0} / d y$ appears on the right hand side in the case of a zonal flow $V_{0}(y)$ sheared latitudinally, and this, of course, may give rise to Kelvin-Helmholtz instabilities.) This exchange term is absent in Paldor's shallow water treatment in which the medium is assumed strictly incompressible. However, I am not convinced that these are crucial differences except, of course, that shallow water theory cannot account for the static instability which arises when $N^{2}<0$. Equation (17), although of obvious physical interest, is redundant since it follows from the equations of motion of the system and is not an independent equation. It is also a rather "ignorant" equation in that it is "oblivious" to the important dynamical action of the Coriolis force (which is perpendicular to $\underline{\mathrm{u}}$ ) and the crucial $\beta$-effect. However the wave energyexchange equation is obviously true at every point of the fluid at all times. Its integral version over an arbitrary surface enclosing a volume is merely the wave perturbation form of the general energy theorem which states that "the total energy of a volume of fluid increases at a rate equal to that at which work is being done on the bounding surface by preasure from without" (Lamb Sir, 1932). Therefore in principle the wave energy equation (either in its differential or integral form) permits the wave energy to change with time and may admit unstable solutions which grow in time contrary to Paldor's claim (Paldor, 2010). In its integral form Paldor applies "suitable bundary conditions" (rigid, free, radiation, evanescent? located where?) to argue away all terms other than the volume integral so as to conclude that $\int_{v} E d \tau=$ const., thereby "proving" that the system cannot possess unstable solutions! As already noted above the wave energy Eq. (17) does not preclude growing solutions. Moreover, as we have have already noted, since the wave energy equation is "oblivious" of the action of Coriolis force, it provides precious little insight into the wave dynamics. Finally as far as the system being in possession of a Poisson bracket and a Hamiltonian is concerned, these properties cannot in any way be interpreted as inconsistent with the predictions of the original equations of motion from which they derive. Therefore Paldor's arguments are not entirely convincing. We emphasize that from the viewpoint of wave dynamics it is well known that if two modes merge or couple in the fashion depicted in the $m>1$ curves of Fig. 1, convective instability ensues Akhiezer et al. (1967).

\section{Conclusions}

In the absence of an obvious source of energy such as the kinetic energy of a sheared zonal flow, it is nevertheless of interest to enquire into the possible energy reservoir which may be tapped to feed this instability. The hydrostatic background state Eq. (4) may be cast in the form

$w_{0}\left(\rho_{0}\right)+g z=$ const

where $w_{0}$ is the enthalpy given by

$w_{0}=\int \frac{d p_{0}}{\rho_{0}}$.

The precise form of $w_{0}\left(\rho_{0}\right)$ depends on the heating/cooling process in the atmosphere, as well as its composition and equation of state. However it is obvious that there exists a negative enthalpy gradient in a similar way that exists in a prescribed temperature gradient which can drive a thermalconvective instability in a fluid heated from below (Chandrasekhar, 1968). Hence enthalpy released by gravitational bouyancy is available to drive an instability provided the system admits of wave modes capable of releasing this latent background energy. In fact the wave dynamic argument leading to the instability condition, $m>1$, may be framed, in terms of the two underlying frequencies of problem, as

$N>2 \Omega\left(\frac{2 \sin ^{2} \theta_{0}}{\cos \theta_{0}}\right) \frac{k_{z} a}{\cos \phi}$

This shows that the Vaisala-Brunt frequency, which measures the strength of the buoyancy force, must be sufficiently greater than the rotational frequency so as to ensure that the wave modes are capable of releasing the latent enthalpy through the action of the gravitational buoyancy force.

This instability is an apparently new and interesting result. Its nonlinear development may play an important role in the atmospheric dynamics of planetary atmospheres. However we emphasize that the instability is of a local nature and that JWKB type solutions may not provide an acceptable solution of the "global" problem. This point requires further study.

Acknowledgements. The author thanks the National Research Foundation (NRF) of South Africa and the Pei-Ling Chair of Physics, UAH, Huntsville, Alabama, USA for their support.

Topical Editor C. Jacobi thanks one anonymous referee for her/his help in evaluating this paper.

\section{References}

Akhiezer, A. I., Akhiezer, I. A., Polovin, R. V., Sitenko, A. G., and Stepanov, K. N.: Collective oscillations in plasma, MIT Press, Cambridge Massachusette, 1967.

Cane, M. A. and Sarachik, E. G.: Forced baroclic ocean motions: I. The linear bounded equation case, J. Mar. Res., 34, 629-665, 1976. 
Chandrasekhar, S.: Hydrodamic and hydromagnetic stability, Clareton Press, Oxford, 1968.

Eckart, C.: Hydrodynamics of ocean and atmospheres, Pergamon Press, Oxford, 1960.

Lamb Sir, H.: "Hydrodynamics", sixth edn., Cambridge University Press, Cambridge, 1932.

Lighthill, M. J.: Waves in fluids, Cambridge University Press, Cambridge, 1980.

McKenzie, J. F.: Instability of coupled gravity-inertial-Rossby waves on a $\beta$-plane in solar system atmospheres, Ann. Geophys., 27, 4221-4227, doi:10.5194/angeo-27-4221-2009, 2009.

McKenzie, J. F.: Instability of combined gravity-inertial-Rossby waves in atmospheres and oceans, Ann. Geophys., 29, 997-1003, doi:10.5194/angeo-29-997-2011, 2011.
Moore, D. and Philander, S. G. H.: The Sea - Modelling of the tropical oceanic circulation, Vol. 6, Wiley-interscience, 1977.

Paldor, N.: On spurious instabilities on the $\beta$-planes with no mean flows, Ann. Geophys., 28, 1737-1739, doi:10.5194/angeo-281737-2010, 2010.

Paldor, N., Rubin, S., and Marian, A. T.: A conscistent theory for linear waves of the shallow water Equations on a rotating plane in mid-latitude, J. Phys-Oceanogr., 39(7), 1685-1699, 2009.

Pedolsky, J.: Waves in ocean and atmosphere: Introduction to wave dynamics, Chapter 13, Springer, 2003. 